\title{
Correction to: Unpaired Image-to-Image Translation Using Adversarial Consistency Loss
}

Yihao Zhao, Ruihai Wu, and Hao Dong

\section{Correction to:}

Chapter "Unpaired Image-to-Image Translation Using Adversarial Consistency Loss" in: A. Vedaldi et al. (Eds.): Computer Vision - ECCV 2020, LNCS 12354, https://doi.org/10.1007/978-3-030-58545-7_46

In the originally published version of this chapter, the acknowledgment section has been modified. 